\title{
ARTICLE OPEN \\ Suicidal ideation and suicide attempts in chronic obstructive pulmonary disease: The Korea National Health and Nutrition Examination Survey (KNHANES IV, V) from 2007-2012
}

\author{
Jae Ho Chung ${ }^{1}$, Chang Hoon Han ${ }^{2}$, Seon Cheol Park ${ }^{2}$ and Cheong Ju Kim ${ }^{2}$
}

BACKGROUND: There is good evidence for an association between chronic obstructive pulmonary disease (COPD) and depression, but there are few studies on the relationship between COPD and suicidal ideation/suicidal attempts.

AIMS: To evaluate the mental health of patients with COPD in Korea and to compare it with that of the general population. METHODS: We analysed data of 15,718 subjects (age $\geqslant 40$ years) who participated in the 2007-2012 Korea National Health and Nutrition Examination Survey. COPD was defined according to the Global Initiative for Chronic Obstructive Lung Disease (GOLD) criteria for people aged $\geqslant 40$ years. We compared the mental health outcomes of 2,506 patients with COPD (2,355 GOLD stages I and II; 151 GOLD stages III and IV) with those of 13,212 controls.

RESULTS: Suicidal thoughts were reported by $16.0 \%$ of patients in stages I and II, by $23.8 \%$ of those in stages III and IV and by $15.7 \%$ of controls $(P=0.023)$. Suicidal attempts were reported by $0.6 \%$ of patients in stages I and II, by $2.6 \%$ of those in stages III and IV and by $1.0 \%$ of controls $(P=0.019)$. The crude odds ratio $(\mathrm{OR})$ for suicidal ideation in those in stages III and IV was 1.68 (95\% confidence interval (Cl), 1.16-2.46), and the OR for suicidal attempts in stages III and IV was 2.83 ( $95 \% \mathrm{Cl}, 1.03-7.75)$. In multivariate analysis, the OR for suicidal ideation in stages III and IV was 1.67 (95\% Cl, 1.12-2.49) and that for suicidal attempts was $2.94(95 \% \mathrm{Cl}, 1.03-8.31)$. CONCLUSIONS: GOLD stages III and IV COPD were associated with a marked increase in suicidal behaviour.

npj Primary Care Respiratory Medicine (2014) 24, 14094; doi:10.1038/npjpcrm.2014.94; published online 30 October 2014

\section{INTRODUCTION}

Chronic obstructive pulmonary disease (COPD) is a leading cause of global morbidity and disability and is predicted to become the third greatest cause of death worldwide by $2020 .^{1}$ Although the underlying pathology is initially confined to the lungs, the associated emotional responses to COPD contribute greatly to the resulting morbidity. Many chronic medical conditions are complicated by emotional and psychological disorders, and yet the emotional dimensions of such chronic medical conditions are often overlooked when medical care is considered. ${ }^{2}$ Studies have consistently documented strong links between COPD and depression., ${ }^{3,4}$ A meta-analysis using 16 studies showed that COPD consistently increased the risk of depression (crude odds ratio (OR), 1.69; 95\% confidence interval (Cl), 1.45-1.96). ${ }^{5}$ Depression and anxiety adversely affect prognosis in COPD, and COPD increases the risk of developing depression. These bidirectional associations suggest the potential usefulness of screening for these disease combinations to direct timely therapeutic intervention. Although a relationship has been established between high levels of depression and COPD, there are few studies on the relationship between COPD and suicidal ideation and attempts., ${ }^{6,7}$ Goodwin $^{6}$ studied the association among COPD, suicidal ideation and suicide attempts and found that COPD was associated with increased odds of suicidal ideation but that the association was no longer significant after adjusting for nicotine dependence. However, suicide attempts were higher in individuals with COPD and remained so after adjusting for demographic factors, depression, panic disorder, drug and alcohol dependence and nicotine dependence. Druss and Pincus ${ }^{7}$ found a significant increase in suicidal ideation in patients with asthma and chronic bronchitis, but not in those with hypertension, arthritis, thyroid disease, diabetes or cancer, after adjusting for major depression, depressive symptoms, heavy alcohol use and demographic factors. Because depression is the strongest risk factor for suicidal thoughts and behaviour, it is logical that a heavy burden of depression among those with COPD could put this group at an increased risk for suicide. Moreover, smoking has also been linked with suicidal behaviour ${ }^{8}$ and is intricately linked with COPD, suggesting further impetus for looking at suicidal behaviour among those with COPD. Suicide is an important public health concern in South Korea and worldwide. The rate of suicide has increased dramatically in Korea, and it is now the fourth leading cause of death. ${ }^{9}$ Epidemiological differences lead to a need to investigate different comorbidities and risk factors in different ethnic groups.

The purpose of this study was to evaluate the mental health of patients with COPD and compare it with that of the general population of Korea.

\section{MATERIALS AND METHODS}

Study participants

This study was based on data from the Fourth Korean National Health and Nutrition Examination Survey (KNHANES IV, 2007-2009) and KNHANES V (2010-2012). KNHANES has been conducted periodically to assess the health and nutritional status of the Korean civilian non-institutionalised population. KNHANES is composed of a health interview survey, a health examination survey and a nutrition survey conducted by trained investigators. Annually, 10,000-12,000 individuals from 4,600 households are selected from a panel to represent Koreans aged $\geqslant 18$ years using the multistage clustered and stratified random sampling method based on

\footnotetext{
'Division of Pulmonology, Department of Internal Medicine, International St. Mary's Hospital, Catholic Kwandong University College of Medicine, Incheon, Republic of Korea and ${ }^{2}$ Division of Pulmonology, Department of Internal Medicine, National Health Insurance Service Ilsan Hospital, Koyang, Republic of Korea.

Correspondence: CH Han (hch7001@nhimc.or.kr)

Received 30 June 2014; revised 26 August 2014; accepted 15 September 2014
} 
National Census Data. The sampling frame was developed on the basis of the 2005 population and housing census in Korea. Household units were selected by a stratified multistage probability sampling design for the South Korean population. KNHANES IV and V were cross-sectional, nationally representative surveys conducted by the Division of Chronic Disease Surveillance, Korea Centers for Disease Control and Prevention, among $\sim 260,000$ primary sampling units, each of which contained $\sim 60$ households. Of the participants, we analysed data on COPD, depression and suicidal ideation from 15,718 subjects aged $\geqslant 40$ years after excluding 1,855 non-respondents.

\section{Lung function measurement}

Spirometry was performed by trained technicians according to the 1994 American Thoracic Society recommendations using the same type of dry rolling-seal spirometer (Model 2130; SensoMedics, Yorba Linda, CA, USA) for all subjects.

Airway obstruction was defined according to the Global Initiative for Chronic Obstructive Lung Disease (GOLD) criteria (GOLD stage I) as forced expiratory volume in $1 \mathrm{~s} /$ forced vital capacity $\left(\mathrm{FEV}_{1} / \mathrm{FVC}\right)<70 \% .^{10}$ Severity of airway obstruction was based on the percentage predicted $\mathrm{FEV}_{1}$ in accordance with the GOLD criteria $\left(\mathrm{FEV}_{1} \geqslant 80 \%\right.$ predicted, stage 1 , mild $\mathrm{FEV}_{1}=50-79 \%$ predicted, stage II, moderate; $\mathrm{FEV}_{1}=30-49 \%$ predicted, stage III, severe; $\mathrm{FEV}_{1}<30 \%$ predicted, stage IV, very severe). The restrictive spirometric pattern was defined as $\mathrm{FEV}_{1} / \mathrm{FVC}>70 \%$ and $\mathrm{FVC}$ $<80 \%$ predicted.

\section{Baseline physical health}

Physical health may affect an individual's mental health and future mortality risk. Comorbid conditions include stroke, hypertension, ischaemic heart disease, liver cirrhosis, arthritis, asthma and chronic renal failure. The number of comorbid conditions was also evaluated. Subjects with one or more cancers of the stomach, liver, lung, colon, cervix or breast were defined as 'major cancer' subjects, based on their answering 'yes' to the question 'Were you diagnosed with cancer(s) by a physician?' to avoid bias generated by a subjective assessment.

\section{Socioeconomic status}

Indicators of socioeconomic status are associated with suicidal ideation. ${ }^{11}$ In the present study, education, occupation and household income were used as indicators of socioeconomic status. Self-reported smoking, alcohol intake and physical activity were estimated from questionnaire responses. Household income was categorised according to the quartile of total income of each member in the household. Marital status was categorised as married, single or divorced/separated/widowed.

\section{Health behaviours}

Suicide-related thoughts and behaviours are associated with health behaviours such as cigarette smoking, ${ }^{12}$ alcohol consumption ${ }^{13}$ and physical exercise. ${ }^{14}$ Health behaviours such as smoking, drinking, physical activity and sleep were assessed through self-reported questionnaires. Smokers were classified as current, former or non-smokers. Individuals were defined as risky drinkers if they reported 12 or more binge drinking episodes (consumption of five or more alcoholic beverages in a single day) during the previous year. ${ }^{15}$

Individuals were defined as having 'physical activity below recommendations' if they did not report engaging in either $20 \mathrm{~min}$ or more of vigorous physical activity three or more times per week or $30 \mathrm{~min}$ or more of light/ moderate physical activity five or more times per week, in keeping with the American College of Sports Medicine Guidelines during the survey period. ${ }^{16}$

\section{Mental health measures}

Psychosocial factors can affect the relationship between suicidal ideation and mortality, and suicidal ideation can also be considered a marker of psychosocial factors. Depression is based on their answering 'yes' to the question 'Were you diagnosed with depression by a physician?'. To assess the mental health of the study population, the surveys provided to the participants included the same questions as those in the KNHANES surveys. Three dimensions within the domains of health status and mental health were determined, such as stress, depression and suicidal thoughts and attempts. Participants reported their level of stress as none, mild moderate or severe. Depression was screened using the Korean version of the World Health Organization Composite International Diagnostic Interview-Short Form, which was validated as a cost-effective screening instrument that could be easily integrated into health surveys. ${ }^{17}$ In your lifetime, have you ever had 2 weeks or more when nearly every day you felt sad, blue or depressed?' and 'Have there ever been 2 weeks or longer when you lost interest in most things such as work or hobbies or things you usually like to do for fun?' To assess depression, participants answered 'yes' or 'no' to a question of whether they had experienced a depressed mood for 2 or more continuous weeks during the previous year. Suicidal ideation was assessed by a positive answer to the question 'In the last 12 months, did you think about committing suicide?' A 'yes' or 'no' response was also used to determine whether they had suicidal thoughts; if they answered 'yes,' they were asked about their suicide attempts, if any.

This indicator is a well-documented predictor of suicide attempts that was previously used in other surveys on adults ${ }^{18}$ and in the KNHANES studies.

\section{Ethical issues}

The institutional review board at the Korea Centers for Disease Control and Prevention approved the study protocol (nos. 2007-02CON-04-P, 200804EXP-01-C, 2009-01CON-03-2C, 2010-02CON-21-C, 2011-02CON-06-C, 2012-01EXP-01-2C), and all participants signed informed consent forms.

\section{Data analysis}

All sampling and weight variables were stratified, and the SPSS survey procedure was used for the statistical analysis to ensure appropriate estimates and standard errors. We used survey sample weights in all analyses to produce non-biased estimates for descriptive or analytical data analysis, Descriptive statistical methods were used to describe the basic characteristics of the study population; numbers and percentages are reported for each variable. Clinical characteristics were compared among participants using analysis of variance for continuous variables and the $x^{2}$ test for categorical variables. Multivariate logistic regression analysis adjusted for age, sex, marital status, smoking, alcohol intake, education, personal income physical activity and presence of diabetes mellitus, hypertension, stress, depressive mood for more than 2 weeks and major cancer (stomach, liver, lung, cervix, breast and colon cancers) was used to examine independent risk factors for suicidal behaviour among patients with COPD. Data were analysed using SPSS for Windows ver. 20.0 (SPSS, Inc., Chicago, IL, USA).

\section{RESULTS}

The baseline characteristics of the study population are described in Table 1. Compared with the general population, patients with GOLD Stages III and IV COPD were more likely to be older, male, less educated, less likely to have a job and more likely to earn a lower income and exhibit a current smoking habit.

Table 2 presents the differences in mental health between the study population and the control group. The proportion of patients with GOLD stages III and IV COPD reporting moderate-tosevere stress was $25.8 \%$ compared with $25.2 \%$ of controls. Depressed mood for 2 or more continuous weeks was reported in $13.4 \%$ of patients with GOLD stages I and II COPD, in $17.6 \%$ of those with GOLD stages III and IV COPD and in $15.1 \%$ of controls $(P=0.063)$. Suicidal thoughts were reported by $16.0 \%$ of patients with GOLD stages I and II COPD, in $23.8 \%$ of those with GOLD stages III and IV COPD and in $15.7 \%$ of controls $(P=0.023)$. Suicide attempts were reported by $0.6 \%$ of patients with GOLD stages I and II COPD, in $2.6 \%$ of those with GOLD stages III and IV COPD and in $1.0 \%$ of controls $(P=0.019)$. The OR for suicidal ideation in those with GOLD stages III and IV COPD was $1.68(95 \% \mathrm{Cl}, 1.16-$ $2.46)$, and the OR for suicidal attempts in that group was $2.83(95 \%$ $\mathrm{Cl}, 1.03-7.75)$. Following adjustment for age, sex, marital status, smoking, alcohol intake, education, personal income, physical activity and presence of diabetes mellitus, hypertension, stress, depressive mood for 2 or more continuous weeks and major cancer, the OR for suicidal ideation in those with GOLD stages III and IV COPD was $1.67(95 \% \mathrm{Cl}, 1.12-2.49)$ and that for suicide attempts was $2.94(95 \% \mathrm{Cl}, 1.03-8.31$; Table 3$)$. 
Table 1. Clinical characteristics of the study populations

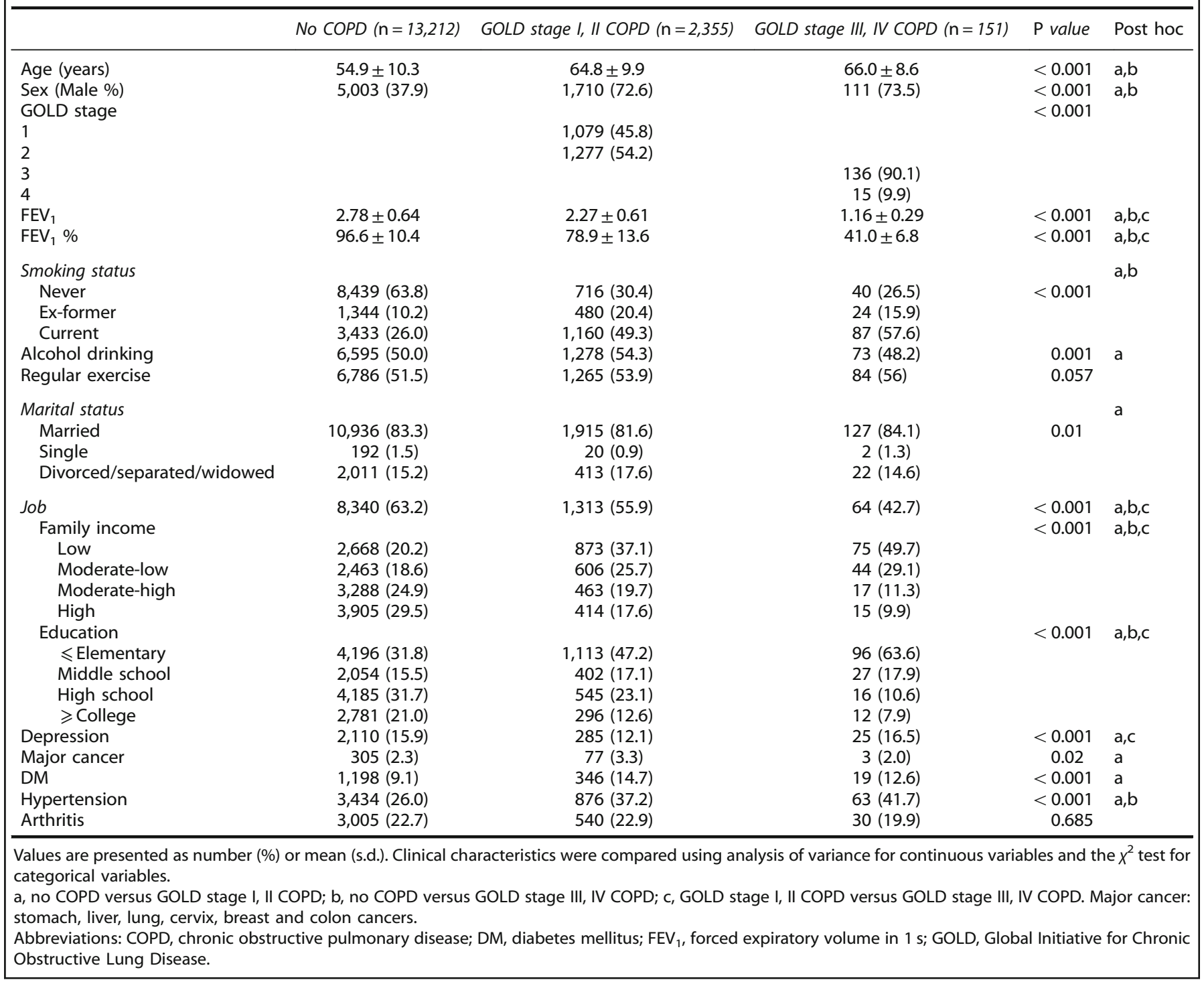

Table 2. Mental health of COPD patients

\begin{tabular}{|c|c|c|c|c|c|}
\hline Stress & & & & $<0.001$ & a \\
\hline None to mild & $9,883(74.8)$ & $1,891(80.3)$ & $112(74.2)$ & & \\
\hline Experiences of depressive mood for 2 or more continuous weeks & $1,994(15.1)$ & 316 (13.4) & 27 (17.9) & 0.063 & a \\
\hline Suicidal thoughts during the previous year & $2,070(15.7)$ & $375(16.0)$ & $36(23.8)$ & 0.023 & $\mathrm{~b}, \mathrm{c}$ \\
\hline Suicidal attempts during the previous year & $126(1.0)$ & $14(0.6)$ & $4(2.6)$ & 0.019 & $\mathrm{~b}, \mathrm{c}$ \\
\hline
\end{tabular}

\section{DISCUSSION}

Main findings

The results of this study suggest that GOLD stages III and IV COPD are significantly associated with an increased likelihood of suicidal ideation and attempts.
Strengths and limitations of this study

The limitations of this study should be considered when evaluating the results. First, assessments of suicidal ideation and suicide attempts occur over a lifetime; therefore, it is not possible to determine the sequence of events, although the distinction 
Table 3. Suicidal behaviours among COPD patients

\begin{tabular}{|c|c|c|c|c|}
\hline & Unadjusted & $\mathrm{P}$ value & Adjusted $^{\mathrm{a}}$ & $\mathrm{P}$ value \\
\hline \multicolumn{5}{|c|}{ GOLD stage I, I/ COPD } \\
\hline $\begin{array}{l}\text { Suicidal } \\
\text { ideation }^{\text {b }}\end{array}$ & $1.02(0.91-1.15)$ & 0.724 & $1.01(0.92-1.12)$ & 0.883 \\
\hline $\begin{array}{l}\text { Suicidal } \\
\text { attempt }\end{array}$ & $1.55(0.91-2.65)$ & 0.109 & $1.41(0.79-2.51)$ & 0.245 \\
\hline \multicolumn{5}{|c|}{ GOLD stage III, IV COPD } \\
\hline $\begin{array}{l}\text { Suicidal } \\
\text { ideation }^{b}\end{array}$ & $1.68(1.16-2.46)$ & 0.007 & $1.67(1.12-2.49)$ & 0.011 \\
\hline $\begin{array}{l}\text { Suicidal } \\
\text { attempt }\end{array}$ & $2.83(1.03-7.75)$ & 0.044 & $2.94(1.03-8.31)$ & 0.044 \\
\hline
\end{tabular}

Abbreviations: COPD, chronic obstructive pulmonary disease; GOLD, Global Initiative for Chronic Obstructive Lung Disease.

a Multivariate logistic analysis, adjusted by age, sex, marital status, smoking, alcohol intake, education, personal income, physical activity, diabetes mellitus, hypertension, stress and depressive mood for more than 2 weeks and the presence of major cancer.

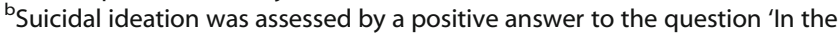
last 12 months, did you think about committing suicide?'

between current and remitted COPD may be helpful in providing more information about the nature of this relationship beyond that available in previous reports. Specifically, we cannot determine whether suicidal thoughts/attempts occurred contemporaneously with COPD or whether a history of suicidal thoughts/attempts preceded COPD. However, because a history of suicidal thoughts/ attempts is the strongest predictor of future behaviour, this information can still be useful to clinicians evaluating potential risk, particularly in the presence of other risk factors for suicidal behaviour. Second, because this was a cross-sectional study, interpretations of a cause-and-effect relationship between suicidal behaviours and related variables are limited. Finally, the limitation of KNHANES is that the subjects who participated in this survey have generally a mild-to-moderate form of comorbidities. Therefore, the small number of COPD patients in stages III and IV would have an influence on the analysis of risk for suicide. Further prospective matched case-control or cohort studies might overcome this limitation. Despite these limitations, our study has important implications for both clinical practice and research. The strength of our study was that the data were obtained from a nationwide population with a high response rate and therefore provide representative information on the Korean population, extensive data on potential confounders, and a large sample size that increased precision and permitted multiple statistical adjustments. Patients with COPD reported greater levels of suicidal thoughts compared with those in the general population. Therefore, it is important for practising general physicians to be aware that patients with COPD are at risk of significant mental health problems. These health-care workers should be encouraged to screen for and promptly diagnose these problems and refer patients to appropriate mental health professionals and services.

Interpretation of findings in relation to previously published work Few studies have been conducted on the prevalence of suicide and suicidal behaviours in patients with COPD. Goodwin ${ }^{6}$ studied the association among COPD, suicidal ideation and suicide attempts and found that COPD was associated with increased odds of suicidal ideation but that the association was no longer significant after adjusting for nicotine dependence. However, suicide attempts were higher in individuals with COPD and remained so after adjusting for demographic factors, depression, panic disorder, drug and alcohol dependence and nicotine dependence. Druss and Pincus ${ }^{7}$ found a significant increase in suicidal ideation in patients with asthma and chronic bronchitis, but not in those with hypertension, arthritis, thyroid disease, diabetes or cancer, after adjusting for major depression, depressive symptoms, heavy alcohol use and demographic factors. With similar adjustments, suicide attempts were seen to increase significantly only in those with asthma $(O R, 4.3)$, chronic bronchitis $(\mathrm{OR}, 2.6)$ and cancer $(\mathrm{OR}, 4.5)$. Goodwin et al. ${ }^{19}$ adjusted for demographic factors and a number of Axis I disorders and found an association between suicide attempts and lung disease (OR, 1.8; 95\% Cl, 1.1-2.7), ulcer (OR, 2.1; 95\% Cl, 1.3-2.4) and AIDS (OR, 44.1; $95 \% \mathrm{Cl}, 10.5-185.6)$, but not arthritis, hypertension, diabetes, heart attack, kidney problems, stroke, autoimmune disease or stomach problems. Webb et $a l^{20}$ found that coronary heart disease, stroke, COPD and osteoporosis were linked to suicide, whereas cancer, hypertension, diabetes, asthma, osteoarthritis, back pain and epilepsy were not. The association with suicide was explained by clinical depression for all conditions except osteoporosis. That study differed from a number of others, including those discussed previously, in finding no association between asthma and suicide and in finding that the association between COPD and suicide was explained by depression. Overall, these studies provide modest support for the idea that impaired lung function may predispose patients to suicide more than do some other physical diseases.

In 2012, South Korea had the highest suicide rate of all countries in the Organization for Economic Cooperation and Development (OECD). In recent years, suicide rates have increased rapidly and steadily in South Korea: in 1990 an average of 9.8 suicides per 100,000 individuals were observed, and in 2012 the rate was 28.1 suicides per 100,000 individuals. ${ }^{21}$ Risk of suicide in those with other chronic diseases in Korea was as follows: diabetes mellitus, OR $2.55(95 \% \mathrm{Cl}, 1.30-5.00)^{22}$; cardiovascular disease, OR $2.95(95 \%$ Cl 1.28-6.81) ${ }^{23}$; and cancer, OR 2.00 (95\% Cl 1.91-2.08). ${ }^{24}$ In our study, the risk of suicidal attempts in GOLD stages III and IV COPD was 2.94 (95\% Cl, 1.03-8.31). The level of risk may be high compared with that of other chronic diseases.

Depression is a common comorbidity in COPD and has a significant impact on patients, their families, society and the course of the disease. ${ }^{3,4}$ Our study showed that depressive symptoms were not different between GOLD stage I/II COPD and GOLD stage III/IV COPD. However, the study by Hanania et al., ${ }^{25}$ consisting of 2,118 COPD patients who were assessed for depression by CES-D (Center for Epidemiologic Studies of Depression) by means of 20 questionnaires, showed that depression symptoms were more prevalent in GOLD stages III and IV COPD compared with GOLD stage II COPD. The inconsistent findings regarding the GOLD COPD stages and depression may be related to methodological differences. The depressive symptoms in our study were assessed by using the answer to a single question 'Have you felt sad or desperate continuously for $\geqslant 2$ weeks so as to disturb your everyday life?' Watkins et al. ${ }^{26}$ investigated the accuracy of a single question 'Do you often feel sad or depressed?' in screening for depression in a cohort of patients after stroke and reported a sensitivity of $86 \%$, a specificity of $78 \%$, a positive predictability of $82 \%$ and a negative predictability of $82 \%$.

Recent research indicates that suicide rates are elevated in those living at higher altitudes, ${ }^{27,28}$ in smokers ${ }^{29,30}$ and in those with asthma. ${ }^{31,32} \mathrm{~A}$ possible mechanism is metabolic stress associated with hypoxia. Young ${ }^{33}$ proposed that low brain serotonin synthesis due to hypoxia may be a factor in the high suicide rates seen in people living at an altitude, in smokers and in patients with COPD and asthma. Pulmonary function deteriorates during disease progression, and the risk for alveolar hypoxia and consequent hypoxemia increases. ${ }^{34}$

Alveolar hypoxia and consequent hypoxemia increase in prevalence as COPD severity increases. This explains why GOLD stages III and IV COPD were significantly associated with increased likelihood of suicidal ideation and attempts among adults compared with those with GOLD stages I and II COPD. 
Implication for future research, policy and practice

These findings support further investigation into the disability and severity of COPD and other common chronic diseases and their links to suicidal ideation and behaviour. Our results may also provide an empirical foundation for including COPD in the clinical assessment of suicide risk, which ultimately may help identify individuals at high risk for suicide. Given that the results from the baseline medical examination provided feedback to the study members, for those participants with a study-detected diagnosis, it is possible that information about their new condition could have precipitated a significant mental health problem, leading to a successful attempt to take their lives.

\section{Conclusion}

Our results add to the growing evidence that severe (GOLD stages III and IV) COPD is associated with increased suicidal ideation and suicide attempts among adults in Korea.

\section{COMPETING INTERESTS}

The authors declare no conflict of interest.

\section{CONTRIBUTIONS}

All authors contributed to the conception, analysis, interpretation, revision and final approval of the manuscript. JHC served as a principal investigator and had full access to all of the data in the study. $\mathrm{CHH}$ takes responsibility for the integrity of the data and for the accuracy of the data analysis. SCP and CJK provided study management.

\section{FUNDING}

The authors declare that no funding was received.

\section{REFERENCES}

1 Murray CJ, Lopez AD. Alternative projections of mortality and disability by cause 1990-2020: Global Burden of Disease Study. Lancet 1997; 349: 1498-1504.

2 Turner J, Kelly B. Emotional dimensions of chronic disease. West J Med 2000; 172: 124-128.

3 Laurin C, Lavoie KL, Bacon SL, Dupuis G, Lacoste G, Cartier A et al. Sex differences in the prevalence of psychiatric disorders and psychological distress in patients with COPD. Chest 2007; 132: 148-155.

4 Schneider C, Jick SS, Bothner U, Meier CR. COPD and the risk of depression. Chest 2010; 137: 341-347.

5 Atlantis E, Fahey P, Cochrane B, Smith S. Bidirectional associations between clinically relevant depression or anxiety and COPD: a systematic review and meta-analysis. Chest 2013; 144: 766-777.

6 Goodwin RD. Is COPD associated with suicide behavior? J Psychiatr Res 2011; 45: 1269-1271.

7 Druss $B$, Pincus $H$. Suicidal ideation and suicide attempts in general medical illnesses. Arch Intern Med 2000; 160: 1522-1526.

8 Hughes JR. Smoking and suicide: a brief overview. Drug Alcohol Depend 2008; 98 169-178.

9 Office KNS (ed). 2010 Annual report on the Cause of Death. Korean National Statistical Office: Seoul, South Korea, 2011.

10 Rabe KF, Hurd S, Anzueto A, Barnes PJ, Buist SA, Calverley P et al. Global strategy for the diagnosis, management, and prevention of chronic obstructive pulmonary disease: GOLD executive summary. Am J Resp Crit Care Med 2007; 176: 532-555.

11 Nock MK, Borges G, Bromet EJ, Alonso J, Angermeyer M, Beautrais A et al. Crossnational prevalence and risk factors for suicidal ideation, plans and attempts. $\mathrm{Br} J$ Psychiatry 2008; 192: 98-105.

12 Kessler RC, Borges G, Sampson N, Miller M, Nock MK. The association between smoking and subsequent suicide-related outcomes in the National Comorbidity Survey panel sample. Mol Psychiatry 2009; 14: 1132-1142.

13 Pfaff JJ, Almeida OP, Witte TK, Waesche MC, Joiner TE Jr. Relationship between quantity and frequency of alcohol use and indices of suicidal behavior in an elderly Australian sample. Suicide Life Threat Behav 2007; 37: 616-626.
14 Brown DR, Galuska DA, Zhang J, Eaton DK, Fulton JE, Lowry R et al. Psychobiology and behavioral strategies. Physical activity, sport participation, and suicidal behavior: U.S. high school students. Med Sci Sports Exerc 2007; 39: 2248-2257.

15 Coups EJ, Ostroff JS. A population-based estimate of the prevalence of behavioral risk factors among adult cancer survivors and noncancer controls. Prev Med 2005. 40: 702-711.

16 Haskell WL, Lee IM, Pate RR, Powell KE, Blair SN, Franklin BA et al. Physical activity and public health: updated recommendation for adults from the American College of Sports Medicine and the American Heart Association. Circulation 2007; 116: $1081-1093$.

17 Gigantesco A, Morosini P. Development, reliability and factor analysis of a selfadministered questionnaire which originates from the World Health Organization's Composite International Diagnostic Interview - Short Form (CIDI-SF) for assessing mental disorders. Clin Pract Epidemiol Ment Health 2008; 4: 8.

18 Gaynes BN, West SL, Ford CA, Frame P, Klein J, Lohr KN. Screening for suicide risk in adults: a summary of the evidence for the U.S. Preventive Services Task Force. Ann Intern Med 2004; 140: 822-835.

19 Goodwin RD, Marusic A, Hoven CW. Suicide attempts in the United States: the role of physical illness. Soc Sci Med 2003; 56: 1783-1788.

20 Webb RT, Kontopantelis E, Doran T, Qin P, Creed F, Kapur N. Suicide risk in primary care patients with major physical diseases: a case-control study. Arch Gen Psychiatry 2012; 69: 256-264.

21 Statistics Korea. Causes of death statistics in 2013. Statistics Korea: Daejeon, Korea, 2014. Available from http://kostat.go.kr.

22 Batty GD, Kivimaki M, Park IS, Jee SH. Diabetes and raised blood glucose as risk factors for future suicide: cohort study of 1234927 Korean men and women. J Epidemiol Community Health 2012; 66: 650-652.

23 Khang YH, Kim HR, Cho SJ. Relationships of suicide ideation with cause-specific mortality in a longitudinal study of South Koreans. Suicide Life Threat Behav 2010; 40: 465-475.

24 Ahn E, Shin DW, Cho SI, Park S, Won YJ, Yun YH. Suicide rates and risk factors among Korean cancer patients, 1993-2005. Cancer Epidemiol Biomarkers Prev 2010; 19: 2097-2105.

25 Hanania NA, Mullerova H, Locantore NW, Vestbo J, Watkins ML, Wouters EF et al. Determinants of depression in the ECLIPSE chronic obstructive pulmonary disease cohort. Am J Resp Crit Care Med 2011; 183: 604-611.

26 Watkins C, Daniels L, Jack C, Dickinson H, van Den Broek M. Accuracy of a single question in screening for depression in a cohort of patients after stroke: comparative study. BMJ 2001; 323: 1159

27 Haws CA, Gray DD, Yurgelun-Todd DA, Moskos M, Meyer L, Renshaw PF. The possible effect of altitude on regional variation in suicide rates. Med Hypotheses 2009; 73: 587-590.

28 Kim N, Mickelson JB, Brenner BE, Haws CA, Yurgelun-Todd DA, Renshaw PF. Altitude, gun ownership, rural areas, and suicide. Am J Psychiatry 2011; 168: 49-54.

29 Aubin HJ, Berlin I, Reynaud M. Current smoking, hypoxia, and suicide. Am J Psychiatry 2011; 168: 326-327.

30 Li D, Yang X, Ge Z, Hao Y, Wang Q, Liu F et al. Cigarette smoking and risk of completed suicide: a meta-analysis of prospective cohort studies. J Psychiatr Res 2012; 46: 1257-1266.

31 Goodwin RD. Asthma and suicide: current knowledge and future directions. Curr Psychiatry Rep 2012; 14: 30-35.

32 Goodwin RD, Demmer RT, Galea S, Lemeshow AR, Ortega AN, Beautrais A. Asthma and suicide behaviors: results from the Third National Health and Nutrition Examination Survey (NHANES III). J Psychiatr Res 2012; 46: 1002-1007.

33 Young SN. Elevated incidence of suicide in people living at altitude, smokers and patients with chronic obstructive pulmonary disease and asthma: possible role of hypoxia causing decreased serotonin synthesis. J Psychiatry Neurosci 2013; 38: 423-426.

34 Vestbo J, Hurd SS, Agusti AG, Jones PW, Vogelmeier C, Anzueto A et al. Global strategy for the diagnosis, management, and prevention of chronic obstructive pulmonary disease: GOLD executive summary. Am J Resp Crit Care Med 2013; 187: 347-365.

(C)(1) This work is licensed under a Creative Commons Attributionther third party material in this article are included in the article's Creative Commons license, unless indicated otherwise in the credit line; if the material is not included under the Creative Commons license, users will need to obtain permission from the license holder to reproduce the material. To view a copy of this license, visit http:// creativecommons.org/licenses/by-nc-sa/4.0/ 